\title{
Engineering Fracture Mechanics
}

\section{On the interaction of channeling/segmentation cracks of coating: Study on the critical spacing}

\author{
Chen-Wu Wu ${ }^{\text {a,b,*, }}$, Guang-Nan Chen ${ }^{\text {a }}$, Kun Zhang ${ }^{\text {a }}$, Nai-Gang Liang ${ }^{\text {a }}$ \\ ${ }^{a}$ Materials Processing Center, Institute of Mechanics, Chinese Academy of Sciences, No. 15 Beisihuanxi Road, Beijing 100080, PR China \\ ${ }^{\mathrm{b}}$ Graduate School of the Chinese Academy of Sciences, Beijing, PR China
}

Received 7 December 2006; received in revised form 21 May 2007; accepted 23 July 2007

Available online 31 July 2007

\begin{abstract}
Channeling/segmentation cracks may arise in the coating subjected to in-plane tensile stress. The interaction between these multiple cracks, say the effect of the spacing between two adjacent cracks on the behaviors of channels themselves and the interface around the interface corners, attracts wide interest. However, if the spacing is greater than a specific magnitude, namely the Critical Spacing (CS), there should be no interaction between such channeling/segmentation cracks. In this study, the mechanism of the effect of the crack spacing on the interfacial stress around the interface corner will be interpreted firstly. Then the existence of the CS will be verified and the relationship between the CS and the so-called stress transfer length in coating will be established for plane strain condition. Finally the dependence of the stress transfer length, simultaneously of the CS, on the sensitive parameters will be investigated with finite element method and expressed with a simple empirical formula.
\end{abstract}

(c) 2007 Elsevier Ltd. All rights reserved.

Keywords: Coating; Channeling/segmentation cracks; Interaction; Critical spacing; Stress transfer length

\section{Introduction}

Films and coatings bonded to substrates often develop in-plane tensile stresses large enough to cause cracking [1]. The craze-cracks always arise in the biaxially tensioned thin elastic films and coatings [2], while parallel channels would develop if the biaxial stress is biased $[3,4]$ as shown in Fig. 1a and b. Herein the investigations on the behaviors of the channels are of particular importance for predicting the fracture process of the films/ coatings. Solutions for a semi-infinite isolated channeling crack in films bonded to a substrate of infinite thickness have been presented by Beuth [5], in which the steady-state energy release rate averaged over the advancing front is expressed as a function of the film thickness, the Young's modulus and Poisson's ratio of the film and Dundur's parameters characterizing the elastic mismatch between the film and the substrate. At the same

\footnotetext{
* Corresponding author. Address: Materials Processing Center, Institute of Mechanics, Chinese Academy of Sciences, No. 15 Beisihuanxi Road, Beijing 100080, PR China. Tel.: +86 1062547527 1; fax: +86 1062545533.

E-mail address: wcw_on@yahoo.com.cn (C.-W. Wu).
} 
time, the interaction among the multiple parallel channels has attracted a lot of attentions $[1,4]$. For instance, the effect of crack spacing on the energy release rate of parallel channeling cracks is particularly investigated by Huang et al [4], of which the results indicate that generally the energy release rate increases as the crack spacing increases.

Recently, particular attentions are also paid to the interaction among those parallel channels already channeled across the film/coating, which are therefore known as segmentation cracks [6] or vertical cracks [7] as depicted in Fig. $1 b$ and c. Such parallel cracks channeling across the film/coating will be named by segmentation cracks all along in this paper. On the one hand, each of these segmentation cracks introduce two crack surfaces in coating and then two interface corners between the films/coatings as shown in Fig. 1c, around which the interfacial stress concentrations arise. Such interfacial stress concentrations are believed to be the origins of the interface damage $[8,9]$. On the other hand, it is commonly accepted that those pre-existing segmentation cracks do increase the strain tolerance of the coating and improve the durability of the coating, which intrigues many research to develop such segmentation cracks by pre-treatments or post-treatments $[6,10]$. Theoretically, Zhou and Kokini [11] study numerically the effect of the segmentation cracks on the behaviors of the interfacial cracks aiming at the thermal barrier coatings, of which the results indicate that during the cooling period following exposure to high temperatures the driving force of the interface crack is obviously influenced by the density of the segmentation cracks. Wu et al. [12] use a plane strain model of a fixed full width to investigate the effect of the uniformly distributed segmentation cracks on the interfacial stresses around the interface corner under the action of in-plane mismatch strain between the coating and

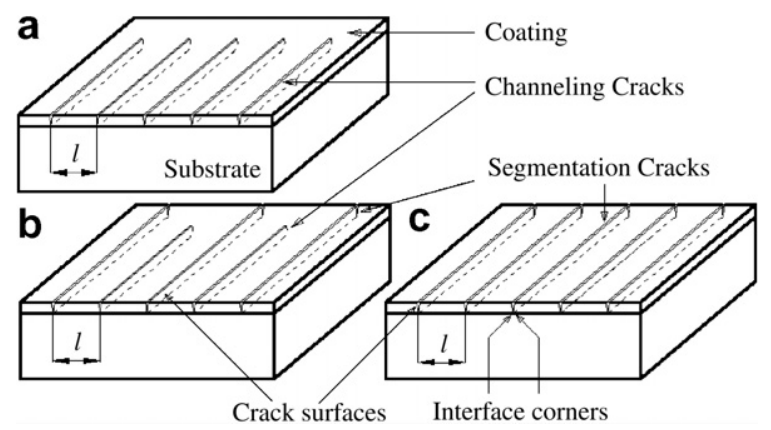

Fig. 1. The schematic of the channeling cracks and segmentation cracks. 
substrate, of which it is found again that the interfacial stresses around the interface corner are prominently influenced by the density of the segmentation cracks, in other words by the spacing between two adjacent segmentation cracks.

Nevertheless, the effect of the crack spacing on either the energy release rate of parallel channeling cracks themselves or the driving force of the interfacial cracks and the interfacial stresses around the interface corners would exist only if the spacing is sufficient small. Because, just as one can easily understand that the crack should not feel the other crack were they a sufficient great distance apart as far as only the loading of the in-plane mismatch strain between coating and substrate is concerned. The minimum magnitude of the spacing between two adjacent cracks required for avoiding the interaction between them may hereby be defined as the Critical Spacing (CS). Such CS is expected to be of finite and determinate for a specific system of coating and substrate, seeking for which will be the main object of this study. And the present work will emphasize particularly on the effect of the spacing between the segmentation cracks on the interfacial stresses around the interface corners, of which the mechanisms account for it and the CS will be particularly demonstrated.

\section{The interpretation on the CS}

\subsection{The stress transfer in segmentation-cracked coating}

Consider a system of segment cracked coating subjected to in-plane tensile stress, say, a uniform temperature increment $\Delta T_{\mathrm{c}}$ in coating relative to the stress-free state, as shown in Fig. 2a, in which the symbols $t_{\mathrm{c}}$ and $t_{\mathrm{s}}$ denote the thicknesses of the coating and substrate, respectively. Each of the segmentation cracks will introduce two crack surfaces in an originally intact coating of infinite dimension in $x$-axis, and then the stress in coating should be released locally [13]. If the spacing between two adjacent cracks, $l$, is sufficient large, the locally redistribution of the stress around one crack should not influence that of another. However, once the spacing $l$ is less than a specific magnitude, that is, the two adjacent cracks are close enough there should be interaction between them. Phenomenologically, in this case, the stress profiles around the interface corners should be influenced by the crack spacing $l$.

The investigations on such systems with different $l$ has been carried out with a symmetrical FEM model as depicted in Fig. 2b, in which the plane strain deformation condition is assumed. To be noted that the symmetry constraints mean the displacements at $X$-direction on both sides of the specimen but the crack face are eliminated. Fig. $3 \mathrm{a}$ and $\mathrm{b}$ presents, respectively, the shear stress $\tau_{x y}$ and normal stress $\sigma_{x x}$ in coating near the crack surfaces for the case $l=100 t_{\mathrm{c}}$, which show a different stress level for every different $y$-ordinate near
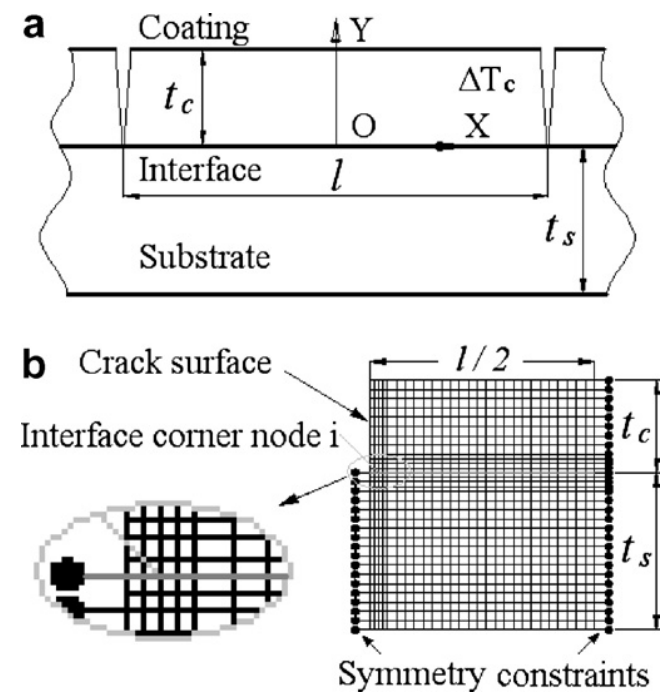

Fig. 2. The schematic of the segment cracked coating and the FEM mesh. 


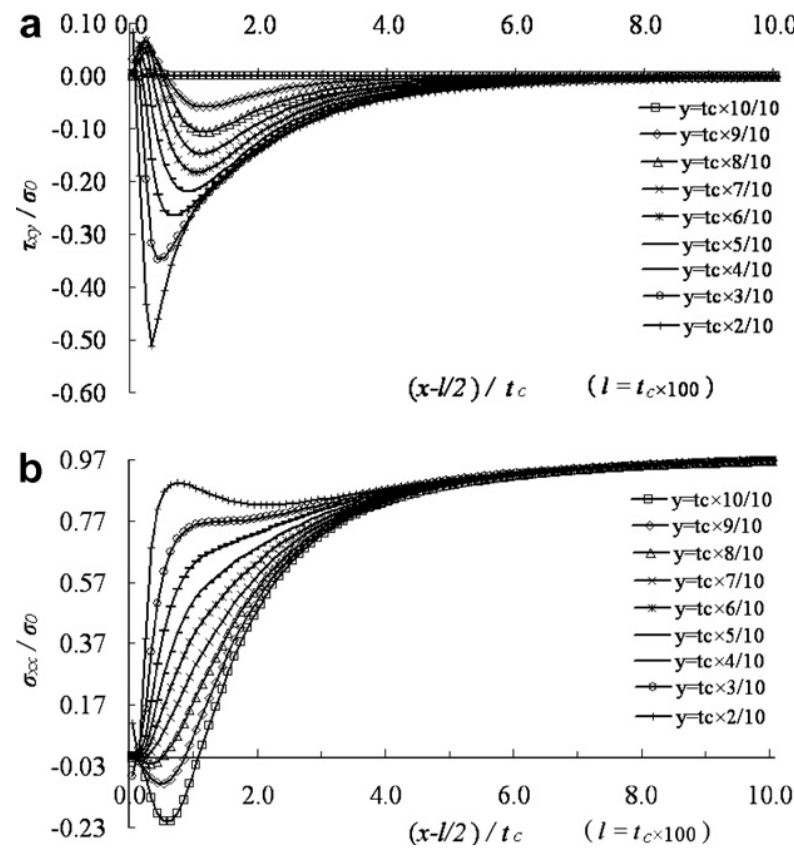

Fig. 3. (a) The shear stress pattern in coating around crack surface for the case $l=100 t_{\mathrm{c}}$. (b) The normal stress pattern in coating around crack surface for the case $l=100 t_{\mathrm{c}}$.

the crack surface. That is, the stress $\tau_{x y}$ and $\sigma_{x x}$ have their non-zero gradients in $y$-direction, i.e. $\frac{\partial \tau_{x y}}{\partial y} \neq 0$ and $\frac{\partial \sigma_{x x}}{\partial y} \neq 0$. However, such gradients vanish away from the crack surface and hereafter the stresses keep uniform within the cross section until reaching their own steady levels. Considering the stress singularity at the interface corner induced by the abrupt change of the elasticity across the interface, the computation stress results of the elements except the nearest two rows from the interface are adopted in this investigation because the region of inaccuracy is limited to about two elements around the interface corner [14]. The average normal stress $\overline{\sigma_{x x}}$ and average shear stress $\overline{\tau_{x y}}$ is herein defined as

$$
\begin{aligned}
& \overline{\sigma_{x x}}=\frac{1}{t_{\mathrm{c}}} \int_{0}^{t_{\mathrm{c}}} \sigma_{x x} \mathrm{~d} y \\
& \overline{\tau_{x y}}=\frac{1}{t_{\mathrm{c}}} \int_{0}^{t_{\mathrm{c}}} \tau_{x y} \mathrm{~d} y
\end{aligned}
$$

These can be approximated with

$$
\begin{aligned}
& \left.\overline{\sigma_{x x}} \doteq \frac{1}{8} \sum_{i=2}^{10} \sigma_{x x}\right|_{y=t_{\mathrm{c}} \times i / 10} \\
& \left.\overline{\tau_{x y}} \doteq \frac{1}{8} \sum_{i=2}^{10} \tau_{x y}\right|_{y=t_{\mathrm{c}} \times i / 10}
\end{aligned}
$$

The results shown in Fig. $4 \mathrm{a}$ and $\mathrm{b}$ indicate that while the average shear stresses $\overline{\tau_{x y}}$ decrease from the negative peak value near the crack surface to zero sharply, the average normal stresses $\bar{\sigma}_{x x}$ in coating increase from zero at crack surface to the maximum magnitude for all the cases $l=100 t_{\mathrm{c}}, 80 t_{\mathrm{c}}, 60 t_{\mathrm{c}}, 40 t_{\mathrm{c}}, 20 t_{\mathrm{c}}, 10 t_{\mathrm{c}}$ and $5 t_{\mathrm{c}}$. While for the cases $l=100 t_{\mathrm{c}}, 80 t_{\mathrm{c}}, 60 t_{\mathrm{c}}, 40 t_{\mathrm{c}}$, a specific distance, which will be named as Stress Transfer Length (STL) 

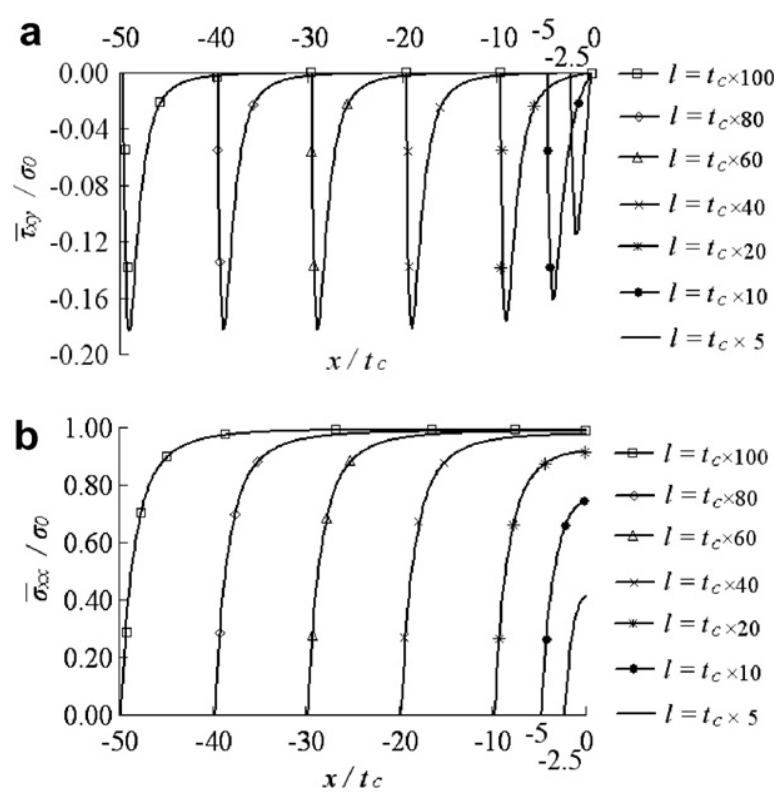

Fig. 4. (a) The average shear stress in coating. (b) The average normal stress in coating.

later, away from the crack surface the maximum magnitudes of the average normal stresses $\overline{\sigma_{x x}}$ maintain a steady level

$$
\sigma_{0}=-E_{\mathrm{c}} \alpha_{\mathrm{c}} \Delta T_{\mathrm{c}} /\left(1-v_{\mathrm{c}}\right)
$$

Herein $E_{\mathrm{c}}, v_{\mathrm{c}}$ represent the elastic modulus and Poisson's ratio of coating, $\alpha_{\mathrm{c}}, \Delta T_{\mathrm{c}}$ denote the coefficients of thermal expansion, temperature increments relative to the stress-free state of the coating. Correspondingly, the average shear stresses $\overline{\tau_{x y}}$ for the cases $l=100 t_{\mathrm{c}}, 80 t_{\mathrm{c}}, 60 t_{\mathrm{c}}, 40 t_{\mathrm{c}}$ reduce to zero at the corresponding locations, at which the average normal stresses $\overline{\sigma_{x x}}$ reach the steady level. The results of the average normal stresses $\overline{\sigma_{x x}}$ in coating shown in Fig. 4b reveal almost the same phenomenon as that of Mezin [13] and Timm et al. [15], that is, the maximum magnitude level would change with the variation of the spacing between two cracks. Nevertheless, to be noted particularly that, above the CS, the change of the spacing does not influence the steady maximum magnitude level, which keeps being $\sigma_{0}$. However, no particular attention has been paid to the existence of the CS before, although some debris of which can be found in the works by Mezin [13] and Timm et al. [15].

To further underline the stress profiles in coating near the crack surfaces, the stresses $\tau_{x y}$ and $\sigma_{x x}$ within more small regions (herein 2.5 times the coating thickness in contrast to 10 times in Fig. 3) for the cases $l=60 t_{\mathrm{c}}$, and $5 t_{\mathrm{c}}$ are depicted in Fig. $5 \mathrm{a}$ and $\mathrm{b}$, and Fig. $6 \mathrm{a}$ and $\mathrm{b}$. One can recognize that the stress profiles for the cases $l=60 t_{\mathrm{c}}$ are almost identical to that of the case $l=100 t_{\mathrm{c}}$ (as shown in Fig. 3a and b), which actually are also identical to that of the cases $l=80 t_{\mathrm{c}}, 40 \mathrm{t}_{\mathrm{c}}, 20 \mathrm{t}_{\mathrm{c}}$ and $10 \mathrm{t}_{\mathrm{c}}$. Although the results of the latter have been omitted to avoid too many pleonastic contents, such identity to the case $l=100 t_{\mathrm{c}}$ can be apparent if only the parts of Fig. 3a and $b$ relevant to that in Figs. 5 and 6 are focused on.

Hereby it can be naturally concluded from the above results that such stress transfer pattern hold for a specific system of coating and substrate if $l / 2$ is greater than the STL $l_{\mathrm{t}}$, namely the least length required before the stress reaches the steady level. This means that if a sequent crack arises at the location a sufficient distance, larger than $2 l_{t}$, apart from the existing crack, the existing crack would have no effect on the local stress field around the crack tip of the sequent crack, i.e., there should be no interaction between such two cracks. That is to say, the sequent channeling crack should not feel the existing crack and behave just like a single one within an originally intact coating were the spacing between two cracks greater than a specific value, namely CS $l_{\mathrm{c}}=2 l_{\mathrm{t}}$. In this case, each of the channels can be treated as an isolate crack and the results by Beuth [5] are applicable to computing the energy release rate of the channels themselves. Once $l$ is less than $l_{\mathrm{c}}=2 l_{\mathrm{t}}$, these channeling cracks will influence each other with disturbing the local stress profiles near the crack surfaces 

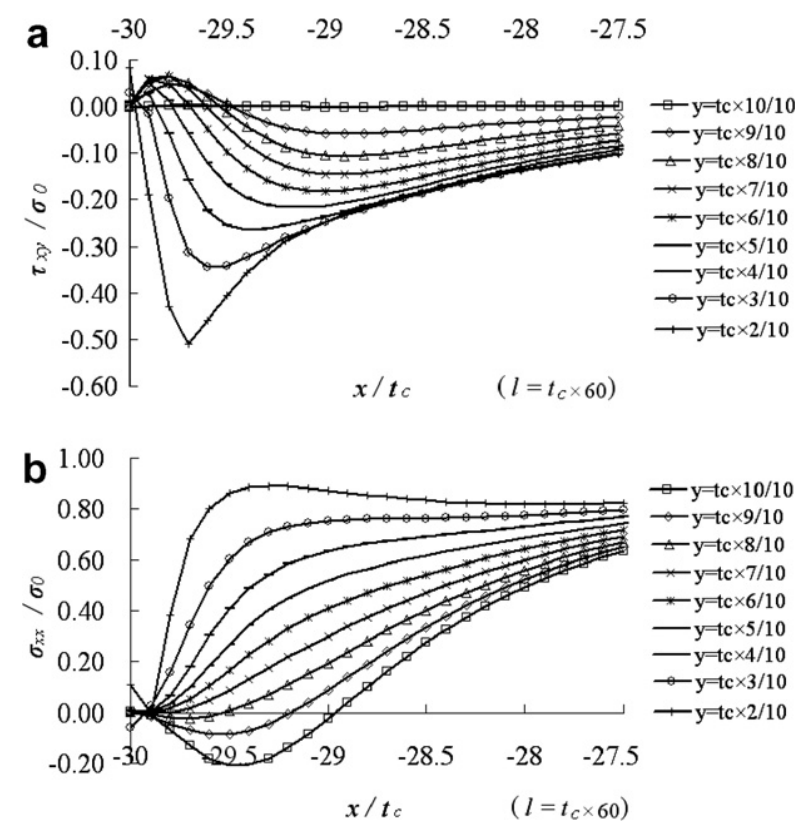

Fig. 5. (a) The shear stress pattern around crack surface for the case $l=60 t_{\mathrm{c}}$. (b) The normal stress pattern around crack surface for the case $l=60 t_{\mathrm{c}}$.

and therefore the solution for an isolate crack should not be applied directly. Thus, to determine the CS for a specific system is of importance for investigating the fracture of the films/coatings themselves.

Note that in these above computations, the materials elastic properties are chosen as, respectively, elastic moduli of the coating and the substrate $E_{\mathrm{c}}=300 \mathrm{GPa}$ and $E_{\mathrm{s}}=200 \mathrm{GPa}$, Poisson's ratios of them $v_{\mathrm{c}}=0.2$ and $v_{\mathrm{s}}=0.255$. And $t_{\mathrm{c}}=0.2 \mathrm{~mm}$ and $t_{\mathrm{s}}=6 \mathrm{~mm}$. At the same time it can be found from the results shown in Figs. $3-5$ that for the system of coating and substrate of the above mechanical and geometrical properties, the CS $l_{\mathrm{c}}$ fall within the range of $20 t_{\mathrm{c}}<l_{\mathrm{c}}<40 t_{\mathrm{c}}$.
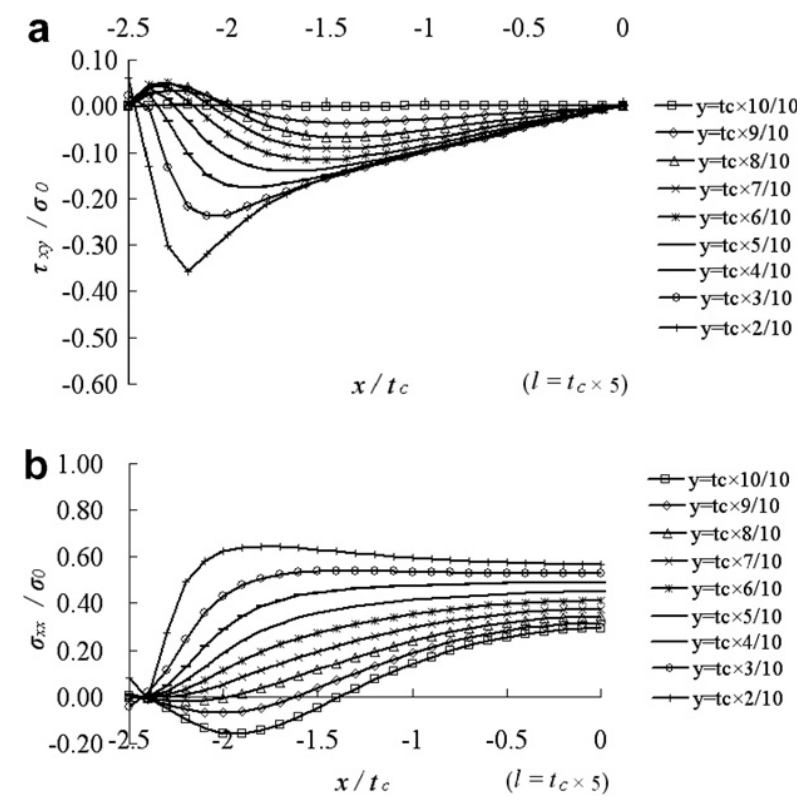

Fig. 6. (a) The shear stress pattern around crack surface for the case $l=5 t_{\mathrm{c}}$. (b) The normal stress pattern around crack surface for the case $l=5 t_{\mathrm{c}}$. 


\subsection{The effect of the spacing on the interfacial stresses and the CS}

The segmentation cracks introduce an interfacial stresses concentration around the interface corner, which is also described with a so-called interface corner stress intensity factor in some literature [8]. Such stress concentration around the interface corner, as aforementioned, is believed to be the origin of the interfacial damage of coating under the action of the in-plane mismatch strain between the files/coatings and substrate, in turn such stress concentration state would be influenced by the spacing between the segmentation cracks according to the work by $\mathrm{Wu}$ et al. [12].

However, our present study shown in Fig. 7 indicates that $l$ influences the interfacial stresses concentration only if $l$ is less than a critical magnitude $l_{\text {ci }}$. The existence of the CS did not be encountered in the work by Wu et al. [12] because the initial maximum width of the specimen had been fixed as a relative small magnitude, which resulted in that the maximum crack spacing was lower than the relevant CS of that system.

In Fig. 7, the stresses of an interface corner node $i$ connecting the second and the third elements from the interface corner are presented considering again the fact that the inaccuracy induced by the stress singularity is limited to about two elements around the interface corner [14]. One can see that both the interfacial shear stress $\tau_{\mathrm{i}}$ and interfacial normal stress $\sigma_{\mathrm{i}}$ keep at the steady maximum level, $\tau_{\text {imax }}$ and $\sigma_{\text {imax }}$ respectively, when $l$ is sufficient great. Actually, this is in consistent with the Saint-Venant principle, because that the influence of a isolate crack under the condition of interest herein can be substituted by a pair of self-equilibrium static forces as shown in Fig. 8. If $l$ is less than $l_{\mathrm{ci}}$, herein some magnitude less than $40 t_{\mathrm{c}}$ while greater than $20 t_{\mathrm{c}}$, the interfacial stresses decrease prominently with the further decrease of $l$, which reveals there must also be a CS $l_{\mathrm{ci}}$ as far as the effect of $l$ on the interface behavior is concerned. Note again that $E_{\mathrm{c}}=300 \mathrm{GPa}$, $E_{\mathrm{s}}=200 \mathrm{GPa}, v_{\mathrm{c}}=0.2, v_{\mathrm{s}}=0.255, t_{\mathrm{c}}=0.2 \mathrm{~mm}$ and $t_{\mathrm{s}}=6 \mathrm{~mm}$ and the plane strain deformation condition are used for these computations. A simple model as illustrated in Fig. 9 is adopted to show that $l_{\mathrm{ci}}=l_{\mathrm{c}}$. In Fig. 9, the static equilibrium of a micro-segment coating is depicted, from which the following relationship can be derived instantly from the equilibrium conditions $\sum X=0$ and $\sum Y=0$ as

$$
\begin{aligned}
\tau_{\mathrm{i}} & =-\frac{\partial N}{\partial x} \\
\sigma_{\mathrm{i}} & =-\frac{\partial S}{\partial x}
\end{aligned}
$$

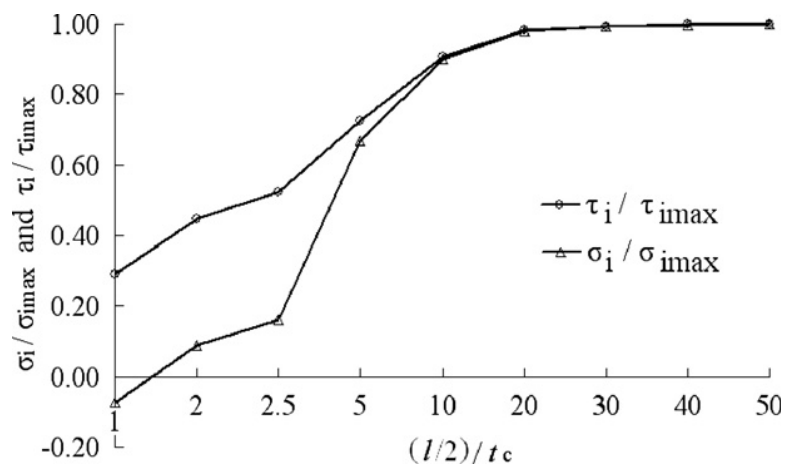

Fig. 7. The influence of $l$ on the interfacial stresses of the node $i$.

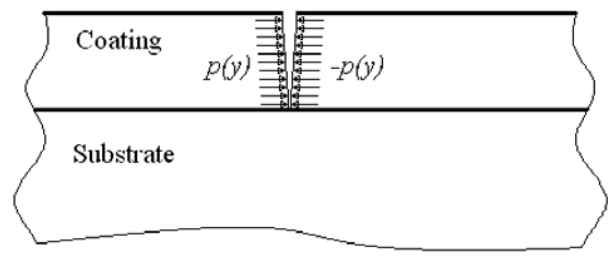

Fig. 8. The self-equilibrium static forces counteracting the effect of crack. 


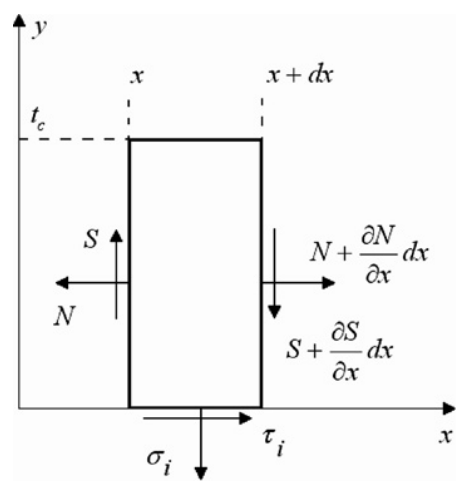

Fig. 9. Static equilibrium of a micro-segment coating subjected to in-plane stress.

for plane strain problem, where

$$
\begin{aligned}
& N=\int_{0}^{t_{\mathrm{c}}} \sigma_{x x} \times 1 \times \mathrm{d} y \\
& S=\int_{0}^{t_{\mathrm{c}}} \tau_{x y} \times 1 \times \mathrm{d} y
\end{aligned}
$$

are, respectively, the resultant normal force and resultant shear force acting on the cross section $X=x$ of unit thickness in $Z$-axis. From Eqs. (5)-(8), one can see that there are determinate relationship between the interfacial stresses and the stresses in coating. That is to say, if the distribution of the stresses in the coating has been fixed on, the distribution of the interfacial stresses shall be determined, vice versus. And once the stresses in the coating approach to a steady state, the interfacial stresses shall disappear. Thus, if $l \geqslant 2 \times l_{\mathrm{t}}$, one crack should not influence the patterns of the stresses $\sigma_{x x}$ and $\tau_{x y}$ near another crack thereafter, not influence the interfacial stresses $\tau_{\mathrm{i}}$ and $\sigma_{\mathrm{i}}$. And if $l<2 \times l_{\mathrm{t}}$, one crack should influence the patterns of the stresses $\sigma_{x x}$ and $\tau_{x y}$ near another crack thereafter, influence the interfacial stresses $\tau_{\mathrm{i}}$ and $\sigma_{\mathrm{i}}$. Hence, $l_{\mathrm{ci}}=2 \times l_{\mathrm{t}}=l_{\mathrm{c}}$ is confirmed concisely.

So far, we have verified the existence of the CS $l_{\mathrm{c}}$, were the spacing between two segmentation cracks above which then one crack would not influence the behaviors of another crack itself or the interfacial stresses around it. Moreover, the relationship between the CS $l_{\mathrm{c}}$ and the STL $l_{\mathrm{t}}$ has been shown to be $l_{\mathrm{c}}=2 l_{\mathrm{t}}$. Thus, the CS $l_{\mathrm{c}}$ can be obtained through computing the STL $l_{\mathrm{t}}$ for a specific system of the coating and substrate.

The concept of STL is originated from the Fiber Reinforced Composites (FRC) and the analyses on it are of popular interest $[16,17]$. Recently, some studies have also been completed to predict the STL in coating $[18,19]$, of which the shear lag model was commonly adopted. Nevertheless, such shear lag model always introduces too many constrains to the application of the results. The present work will be dealt with by FEM to investigate the effect of the sensitive parameters on the STL in coating and search an empirical formula to predict the STL.

\section{The STL}

\subsection{The formulation of the problem}

On assuming the behaviors of both coating and substrate are elastic and isotropic, and the interface between the coating and substrate is perfect everywhere, the STL $l_{\mathrm{t}}$ in coating subjected to in-plane stress would be a function only of $t_{\mathrm{c}}, t_{\mathrm{s}}, E_{\mathrm{c}}, v_{\mathrm{c}}, E_{\mathrm{s}}$ and $v_{\mathrm{s}}$. For the plane strain condition, it can be simplified further as

$$
l_{\mathrm{t}}=f\left(t_{\mathrm{c}}, t_{\mathrm{s}}, \overline{E_{\mathrm{c}}}, \overline{E_{\mathrm{s}}}\right)
$$


where $\overline{E_{\mathrm{c}}}=E_{\mathrm{c}} /\left(1-v_{\mathrm{c}}^{2}\right)$ and $\overline{E_{\mathrm{s}}}=E_{\mathrm{s}} /\left(1-v_{\mathrm{s}}^{2}\right)$. By dimensional analysis with applying I-theorem [20], we obtain

$$
\frac{l_{\mathrm{t}}}{t_{\mathrm{c}}}=\prod\left(\frac{t_{\mathrm{c}}}{t_{\mathrm{s}}}, \overline{\overline{E_{\mathrm{c}}}}\right)
$$

This reveals that the relative STL $l_{\mathrm{t}} / t_{\mathrm{c}}$ should be a function of the ratio of $t_{\mathrm{c}}$ to $t_{\mathrm{s}}$ and that of $\overline{E_{\mathrm{c}}}$ to $\overline{E_{\mathrm{s}}}$. So the relationship between the relative STL and the sensitive parameters will be investigated intensively in what follows.

\subsection{Analyses on the sensitive parameters}

The FEM model is the same as that shown in Fig. 2b, of which $l$ is sufficiently great to ensure the occurrence of the steady stress level $\sigma_{0}$. Seven groups of the ratios of the coating thickness to substrate thickness $t_{\mathrm{c}} / t_{\mathrm{s}}$, $0.5 / 60,1 / 60,2 / 60,3 / 60,4 / 60,5 / 60$ and $6 / 60$ are used to investigate their effect. Corresponding to every case of a specific thickness ratio, six groups of the elasticity ratios $\overline{E_{\mathrm{c}}} / \overline{E_{\mathrm{s}}}, 0.5,1.0,1.5,2.0,2.5$ and 3.0 are used for computation. The results for such 42 cases of different $t_{\mathrm{c}} / t_{\mathrm{s}}$ or $\overline{E_{\mathrm{c}}} / \overline{E_{\mathrm{s}}}$ are illustrated in Fig. 10, in which the curves show the dependence of the $l_{\mathrm{t}} / t_{\mathrm{c}}$ on the ratio $\overline{E_{\mathrm{c}}} / \overline{E_{\mathrm{s}}}$ for different $t_{\mathrm{c}} / t_{\mathrm{s}}$. One can see that the relative STL $l_{\mathrm{t}} / t_{\mathrm{c}}$ increase with the increase of the ratio $\overline{E_{\mathrm{c}}} / \overline{E_{\mathrm{s}}}$, which is easily to accept that a longer length is needed to draw a films/coatings of more rigidity to a prescribed strain level. It is also indicated in Fig. 10 that the relative STL $l_{\mathrm{t}} / t_{\mathrm{c}}$ decrease obviously with the increase of the ratio $t_{\mathrm{c}} / t_{\mathrm{s}}$.

\subsection{The empirical formula of the $S T L$ and the $C S$}

After the effects of the sensitive parameters, $r_{\mathrm{t}}=t_{\mathrm{c}} / t_{\mathrm{s}}$ and $r_{\mathrm{E}}=\overline{E_{\mathrm{c}}} / \overline{E_{\mathrm{s}}}$, on the STL $l_{\mathrm{t}}$ have been obtained as presented above, a simple empirical formula to predict the magnitude of $l_{\mathrm{t}}$ should be of utility. On studying the results as shown in Fig. 10, a diagram of $\ln \left(l_{\mathrm{t}} / t_{\mathrm{c}}\right)$ versus $\ln \left(r_{\mathrm{E}} / r_{\mathrm{t}}\right)$ is produced as shown in Fig. 11, in the latter a property of good linear current appears. Then the dependence of $l_{\mathrm{t}}$ on $r_{\mathrm{t}}$ and $r_{\mathrm{E}}$ can be simply assumed as

$$
\frac{l_{\mathrm{t}}}{t_{\mathrm{c}}}=k_{1}\left(\frac{r_{\mathrm{E}}}{r_{\mathrm{t}}}\right)^{k_{2}}
$$

The constant $k_{1}=\mathrm{e}^{c}$, where $c=\left.\ln \left(l_{\mathrm{t}} / t_{\mathrm{c}}\right)\right|_{\ln \left(r_{\mathrm{E}} / r_{\mathrm{t}}\right)=0}$ is the intercept at $y$-axis by the current line. While $k_{2}=\frac{\ln \left(l_{\mathrm{t}} / t_{\mathrm{c}}\right)}{\ln \left(r_{\mathrm{E}} / r_{\mathrm{t}}\right)}$, which is the slope of the current line shown in Fig. 11. Finally, the empirical formula (11) can be expressed roughly as

$$
\frac{l_{\mathrm{t}}}{t_{\mathrm{c}}} \doteq \mathrm{e}^{1.31} \times\left(\frac{r_{\mathrm{E}}}{r_{\mathrm{t}}}\right)^{0.38} \doteq 3.71 \times\left(\frac{r_{\mathrm{E}}}{r_{\mathrm{t}}}\right)^{0.38}
$$

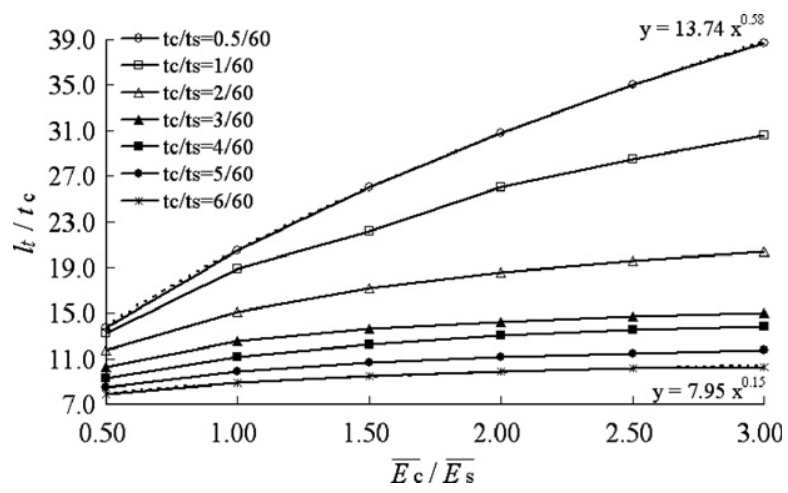

Fig. 10. The relative STL $l_{\mathrm{t}} / t_{\mathrm{c}}$ versus the ratio $\overline{E_{\mathrm{c}}} / \overline{E_{\mathrm{s}}}$ for different $t_{\mathrm{c}} / t_{\mathrm{s}}$. 


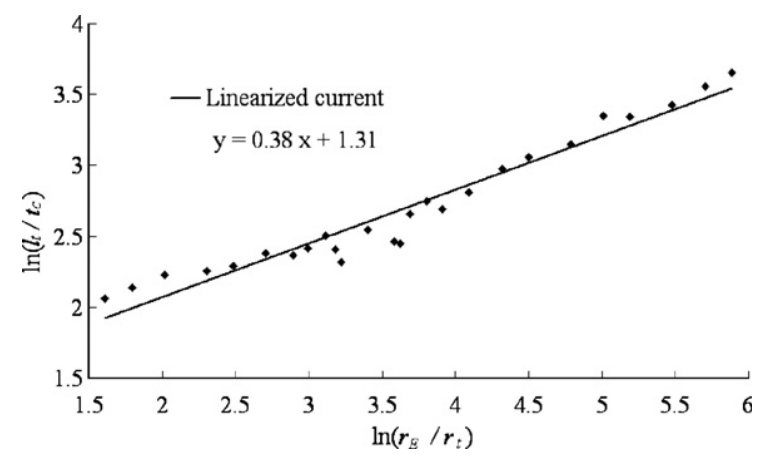

Fig. 11. The empirical formula of $l_{\mathrm{t}} / t_{\mathrm{c}}$ versus $r_{\mathrm{E}} / r_{\mathrm{t}}$.

Thus, the CS $l_{\mathrm{c}}=2 l_{\mathrm{t}}$ can be expressed instantly as

$$
l_{\mathrm{c}} \doteq 7.42 t_{\mathrm{c}} \times\left(\frac{r_{\mathrm{E}}}{r_{\mathrm{t}}}\right)^{0.38}
$$

For instance, when $E_{\mathrm{c}}=300 \mathrm{GPa}, E_{\mathrm{s}}=200 \mathrm{GPa}, v_{\mathrm{c}}=0.2, v_{\mathrm{s}}=0.255, t_{\mathrm{c}}=0.2 \mathrm{~mm}$ and $t_{\mathrm{s}}=6 \mathrm{~mm}$, then $r_{\mathrm{E}} \doteq 1.461, r_{\mathrm{t}}=1 / 30$ and $l_{\mathrm{c}} \doteq 32 t_{\mathrm{c}}$, which indicates that if the spacing between two segmentation cracks there should be no interactions between the segmentation cracks, i.e. neither the stress patterns in coating near the crack surface nor the interfacial stresses around the interface corner should not be influenced by the variation of the spacing. This results is consistent with that mentioned in above sections, which predicted that the CS is within the range of $20 t_{\mathrm{c}}<l_{\mathrm{c}}<40 t_{\mathrm{c}}$.

It should also be noted that the above empirical formula is obtained when the mechanical and geometrical parameters are, respectively, within the ranges of $0.25 / 60 \leqslant r_{\mathrm{t}} \leqslant 6 / 60$ and $0.5 \leqslant r_{\mathrm{E}} \leqslant 3.0$, beyond which the extrapolate prediction should be used cautiously.

\section{Conclusions}

The channeling/segmentation crack should not feel the existence of another one if the spacing between them, $l$, is greater than the CS $l_{\mathrm{c}}$. Reversely, once the spacing $l$ is less than $l_{\mathrm{c}}$, there should be interaction between two channeling/segmentation cracks. The local stress patterns near the crack surfaces of a channeling/segmentation crack are influenced by another one, and therefore the behaviors of the channeling cracks themselves or the interface stresses around the interface corners should be influenced prominently. It was verified again that the interfacial stresses around the interface corner decrease evidently with the further decrease of the spacing $l$ after $l<l_{\mathrm{c}}$.

The determinate relationship was found to exist between the CS $l_{\mathrm{c}}$ and $l_{\mathrm{t}}$, the STL for a specific system of coating and substrate subjected to in-plane mismatch strain, that is $l_{\mathrm{c}}=2 l_{\mathrm{t}}$. Thus, the CS can be achieved through computing the STL for a specific system of coating and substrate.

For a system of the elastic and isotropic film/coating and substrate, the STL $l_{\mathrm{t}}$ is a function only of $r_{\mathrm{t}}=t_{\mathrm{c}} / t_{\mathrm{s}}$ and $r_{\mathrm{E}}=\overline{E_{\mathrm{c}}} / \overline{E_{\mathrm{s}}}$, for which simple empirical formula was established as $\frac{l_{\mathrm{t}}}{t_{\mathrm{c}}} \doteq 3.71 \times\left(\frac{r_{\mathrm{E}}}{r_{\mathrm{t}}}\right)^{0.38}$ when the thickness ratio $r_{\mathrm{t}}$ is in the range of $0.25 / 60 \leqslant r_{\mathrm{t}} \leqslant 6 / 60$ and the elasticity ratio $r_{\mathrm{E}}$ in the range of $0.5 \leqslant r_{\mathrm{E}} \leqslant 3.0$. Therefore the empirical formula for the CS is $l_{\mathrm{c}} \doteq 7.42 t_{\mathrm{c}} \times\left(\frac{r_{\mathrm{E}}}{r_{\mathrm{t}}}\right)^{0.38}$ under the same conditions.

\section{Acknowledgement}

The financial support provided by the National Natural Science Foundation of China (Grant No. 50471087, 50531060 and 10572140) is greatly acknowledged. 


\section{References}

[1] Xia ZC, Hutchinson JW. Crack patterns in thin films. J Mech Phys Solids 2000;48:1107-31.

[2] Parker AP. Stability of arrays of multiple edge cracks. Engng Fract Mech 1999;62:577-91.

[3] Hutchinson JW, Suo Z. Mixed mode cracking in layered materials. Adv Appl Mech 1992;29:3-191.

[4] Huang R, Prevost JH, Huang ZY, Suo Z. Channel-cracking of thin films with the extended finite element method. Engng Fract Mech 2003;70:2513-26.

[5] Beuth JL. Cracking of thin bonded films in residual tension. Int J Solids Struct 1992;29:1657-75.

[6] Guo HB, Vaßen R, Stover D. Atmospheric plasma sprayed thick thermal barrier coatings with high segmentation crack density. Surf Coat Technol 2004;186:353-63.

[7] Madhwal M, Jordan EH, Gell M. Failure mechanisms of dense vertically cracked thermal barrier coatings. Mater Sci Engng A 2004;384:151-61.

[8] Akisanya AR, Meng CS. Initiation of fracture at the interface corner of bi-material joints. J Mech Phys Solids 2003;51:27-46.

[9] Wu CW, Chen GN, Zhang K, Luo GX. A model of interfacial edge fatigue on the failure of thermal protective coatings in bore. Acta Armamentarii (in Chinese) 2006;27(6A):1-4

[10] Batista C, Portinha A, Ribeiro RM, Teixeira V, Costa MF, Oliveira CR. Morphological and microstructural characterization of laser-glazed plasma-sprayed thermal barrier coatings. Surf Coat Technol 2006;200:2929-37.

[11] Zhou B, Kokini K. Effect of pre-existing surface crack morphology on the interfacial thermal fracture of thermal barrier coatings: a numerical study. Mater Sci Engng A 2003;348:271-9.

[12] Wu CW, Chen GN, Zhang K, Luo GX, Liang NG. The effect of periodic segmentation cracks on the interfacial debonding: study on interfacial stresses. Surf Coat Technol 2006;201:287-91.

[13] Mezin A. An analytical solution for stress relaxation in cracked coatings. Surf Coat Technol 2003;166:160-6.

[14] Whitcomb JD, Raju IS, Goree JG. Reliability of the finite element method for calculating free edge stresses in composite laminates. Comput Struct 1982;15:23-37.

[15] Timm DH, Guzina BB, Voller VR. Prediction of thermal crack spacing. Int J Solids Struct 2003;40:125-42.

[16] Cox HL. The elasticity and strength of paper and other fibrous materials. Br J Appl Phys 1952;3:72-9.

[17] $\mathrm{Wu} \mathrm{W,} \mathrm{Verpoest} \mathrm{I,} \mathrm{Varna} \mathrm{J.} \mathrm{An} \mathrm{improved} \mathrm{analysis} \mathrm{of} \mathrm{the} \mathrm{stresses} \mathrm{in} \mathrm{a} \mathrm{single-fibre} \mathrm{reagmentation} \mathrm{test-II.} \mathrm{3-phase} \mathrm{model.} \mathrm{Compos} \mathrm{Sci}$ Technol 1998;58:41-50.

[18] Schadler L, Noyan IC. Quantitative measurement of the stress transfer function in nickel/polyimide thin film/copper thin film structures. Appl Phys Lett 1995;66(1):22-4.

[19] Yanaka M, Kato Y, Tsukahara Y, Takeda N. Effects of temperature on the multiple cracking progress of sub-micron thick glass films deposited on a polymer substrate. Thin Solid Films 1999;355-356:337-42.

[20] Sedov LI. Similarity and dimensional methods in mechanics (Transl.). London: Infosearch; 1959. 